\title{
Effects of Radiation on the Structure of Polypropylene
}

\author{
C. S. WANG* and G. S. Y. YEH \\ Macromolecular Science and Engineering Program, \\ Department of Chemical Engineering, \\ The University of Michigan, Ann Arbor, \\ Michigan 48109, U.S.A.
}

(Received October 20, 1980)

\begin{abstract}
The effects of high-energy radiation on the physical arrangement of macromolecules in atactic polypropylene (aPP) and radiation-amorphized isotactic polypropylene (iPP) were examined using the differential radial distribution function (DRDF) technique. The resultant DRDF curve for $0 \mathrm{Mrad}$ aPP showed three intermolecular peaks at $6.6,13.0$, and $17.7 \AA$ and two intramolecular peaks at 1.5 and $2.5 \AA$. The appearance of the periodic intermolecular DRDF peaks suggested the packing of parallel chains of $\sim 18 \AA$ in the polymer. The $17.7 \AA$ peak disappeared when the aPP sample was irradiated to $115 \mathrm{Mrad}$, indicating the occurrence of structure deterioration from $18 \AA$ to $13 \AA$. As a result of severe deterioration of the intermolecular organization, DRDF curves for 400 and 1300 Mrad iPP samples each showed only one intermolecular peak at $\sim 7 \AA$. This deterioration was attributed to the crosslinking-induced increase in lattice distortions. The results were compared with previous studies on irradiation of natural rubber (NR) and polyethylene (PE). It appears that radiation was more effective in causing deterioration of the intermolecular organization in polypropylene than in NR and PE. This was explained by the different radiation chemistry of the polymers.

KEY WORDS Differential Radial Distribution Function / Wide-Angle XRay Scattering / Polypropylene / Crosslinking / Degradation / Local Structure /
\end{abstract}

It is known that polypropylene (PP) undergoes both crosslinking and degradation in comparable amounts under high-energy irradiation. ${ }^{1}$ This behavior is different from that of typical crosslinking polymers such as natural rubber (NR) and polyethylene (PE), in which radiation causes crosslinking predominantly. ${ }^{2,3}$ Previous studies for determining the amount of radiation-induced crosslinks and chain fractures in PP were reviewed by Geymer. ${ }^{1}$ It appears that the yield of degradation of PP is high at low doses and decreases with increasing irradiation. The temperature of irradiation is also important. At room temperature gelation takes place when the partially degraded PP is irradiated sufficiently. A rapid decrease in the extent of crosslinking is observed at irradiation temperatures above $120^{\circ} \mathrm{C}$.

* Present address: Polymers Department, General Motors Research Laboratories, Warren, Michigan 48090, U.S.A.
The effects of radiation-induced crosslinking on the structure of NR and radiation-amorphized PE have been examined recently at this laboratory. ${ }^{4-6}$ The polymer structures were studied using the differential radial distribution function (DRDF) derived from wide-angle X-ray scattering (WAXS) data. The results suggest that local structures existing in amorphous polymers deteriorate to various degrees at relatively high irradiation doses. For NR, local structures of $\sim 15 \AA$ were found in both unirradiated and irradiated samples up to 550 Mrad. The radiation-induced deterioration occurred at doses higher than $300 \mathrm{Mrad}$ as indicated by the decrease in intermolecular DRDF peak areas. For amorphous PE, deterioration was indicated by radiation-induced size decrease in local structures. The DRDF results showed that local structures of $\sim 25 \AA$ changed to $15 \AA$ when $\mathrm{PE}$ was irradiated from $400 \mathrm{Mrad}$ to $1000 \mathrm{Mrad}$. This structure deterioration was attributed to the crosslinking-induced 
increase in lattice distortions in highly irradiated amorphous polymers.

Thus an examination was made on structure changes brought about by crosslinking and accompanied by substantial degradation during irradiation of the polymers. The present paper describes such a study which was carried out using unirradiated and irradiated atactic polypropylene (aPP) and radiation-amorphized isotactic polypropylene (iPP). Also, this study aims at bringing about a better understanding of the physical arrangement of macromolecules in amorphous polymers. DRDF curves for PP samples were derived from WAXS data using the same technique as reported previously. ${ }^{7}$ The effects of radiation on the structure of amorphous PP are then discussed in terms of a comparison of resultant intermolecular DRDF peaks.

\section{EXPERIMENTAL AND ANALYSIS}

In this study, samples were compression molded from aPP $\left(99 \%\right.$ atactic, $M_{w}=2.0 \times 10^{5}, M_{w} / M_{n}=$ $1.3)$ and $\operatorname{iPP}\left(M_{w}=5.5 \times 10^{5,} M_{w} / M_{n}=7.0\right)$ supplied by Hercules. Two aPP samples, one unirradiated and one irradiated at room temperature to $115 \mathrm{Mrad}$ by ${ }^{60} \mathrm{Co}$ source, were studied. The irradiated aPP sample was sticky and difficult to handle, causing the WAXS data for the $115 \mathrm{Mrad}$ sample less accurate than that of the others studied.

Sauer and coworkers ${ }^{8}$ showed that high-energy radiation causes destruction of crystallinity of iPP. The irradiation was accompanied by density changes in the polymers. ${ }^{8,9}$ In this study the molded iPP samples were irradiated at the University of Michigan reactor at an ambient temperature of approximately $70^{\circ} \mathrm{C}$. The measured densities of iPP samples are shown in Figure 1 as a function of irradiation dose. The density decreases at low irradiation doses but increases after the polymer becomes entirely amorphous, as is consistent with the results in the literature. ${ }^{8,9}$ For the DRDF analysis, amorphous iPP samples of 400 and 1300 Mrad were used. The samples covered a considerably wide range of irradiation for iPP, as is evident from the density data given in Figure 1.

The procedures employed for the WAXS data collection and analysis are described in detail elsewhere. ${ }^{7}$ The X-ray scattering measurements were made on a Philips Norelco diffractometer, using

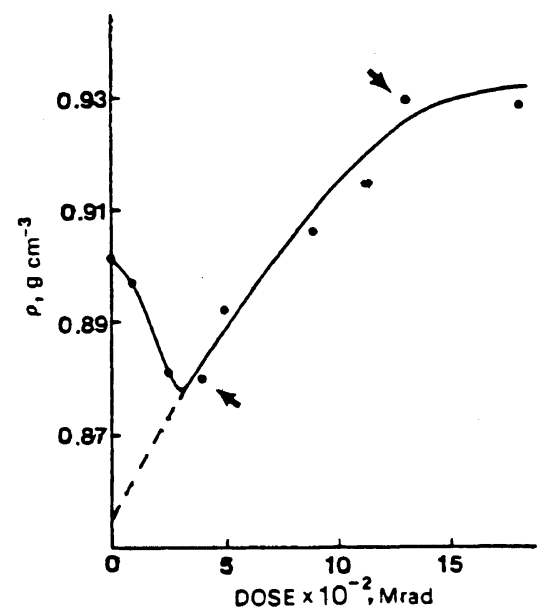

Figure 1. Influence of the dose of reactor radiation on the density of isotactic polypropylene.

$\mathrm{Cu}-K \alpha$ radiation. The radiation was monochromatized by a curved graphite monochromator in the diffraction position. The WAXS intensity for each sample was recorded by a step scanning procedure in the range of 6 to 145 degrees $2 \theta$. Intensities for the lower scattering angles, i.e., $2 \theta<6^{\circ}$, were obtained by extrapolating the experimental curve smoothly to zero scattering angle.

The raw intensity data were corrected for experimental factors, including background air scattering, absorption, polarization, and multiple scattering, according to standard procedures. ${ }^{10-12}$ The corrected intensity data were normalized using the conservation of energy method, ${ }^{11,12}$ and the incoherent Compton scattering was then subtracted. Moreover, PP was treated as a monatomic system. A hypothetical scatterer, $\mathrm{CH}_{2}$, was defined by taking $1 / 3$ of the chemical structural unit of PP.

The resultant DRDF, $4 \pi r^{2} \bar{\rho} H(r)$, for each sample was calculated from the X-ray data by Fourier Transformation,

$$
\begin{aligned}
4 \pi r^{2} \bar{\rho} H(r) & =\frac{2 r}{\pi} \int_{0}^{s_{\max }} s i(s) \sin s r \mathrm{~d} s \\
i(s) & =\frac{I_{\text {coh }}}{f^{2}}-1
\end{aligned}
$$

where $\bar{\rho}$ is the average atomic density, $s=$ $(4 \pi / \lambda) \sin \theta, \quad I_{\text {coh }}$ is the experimental coherent intensity in electron units per scatterer, and $f$ is the scattering factor. The upper bound of the inte- 
gration $s_{\max }$ was $16 \AA^{-1}$. In addition, a final smoothing beyond the second peak of the resultant DRDF was made by multiplying a damping factor, $\exp \left(-0.04 s^{2}\right)$, by the interference function $i(s)$. Possible errors in the available scattering factors were eliminated by adjusting the DRDF curve below $\simeq 1 \AA$ to a straight line whose theoretically expected slope was $-4 \pi \bar{\rho}$.

\section{RESULTS}

The corrected and normalized WAXS patterns for aPP samples are shown in Figure 2. As most amorphous polymers, three diffuse peaks are present at $\sim 16,42$, and 75 degrees $2 \theta$ in each pattern. An $18 \%$ decrease in intensity of the principal peak $\left(16^{\circ}\right)$ is seen in the WAXS pattern of $115 \mathrm{Mrad}$ aPP, compared with that of the $0 \mathrm{Mrad}$ sample. The $1 \%$ stereo-regular component in the unirradiated atactic sample may have caused a trace of crystallinity
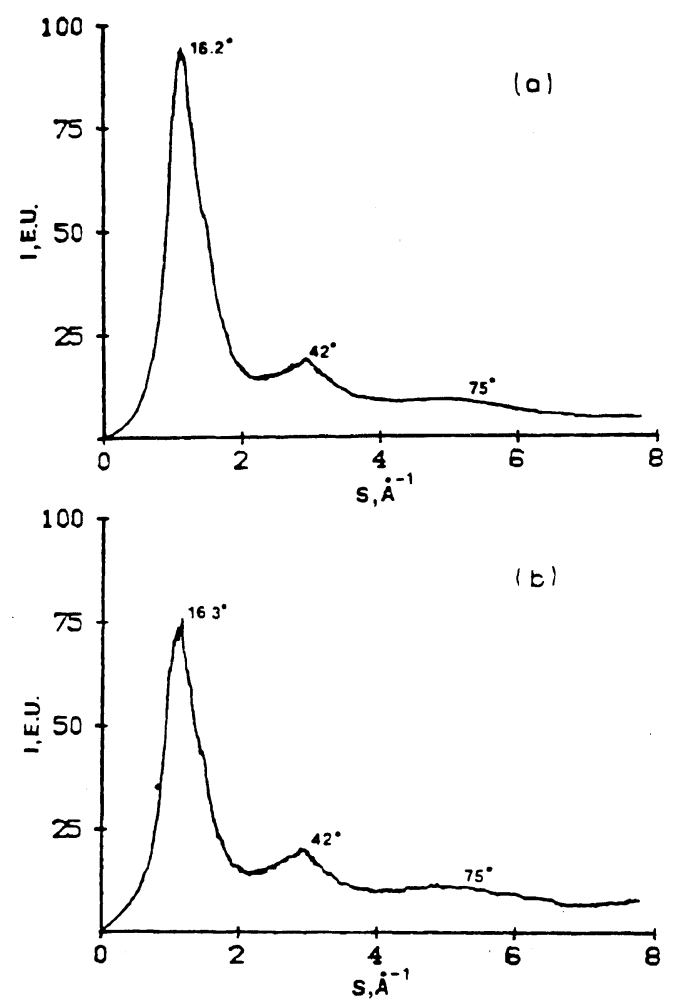

Figure 2. Corrected and normalized experimental WAXS data for (a) $0 \mathrm{Mrad}$ and (b) $115 \mathrm{Mrad}$ atactic polypropylene. The intensity data were normalized by the conservation of energy method. which was too low to be detected by X-rays.

Similarly obtained WAXS patterns for 400 and 1300 Mrad iPP samples are shown in Figure 3. Three intensity peaks are seen at $\sim 16,41$, and 75 degrees $2 \theta$ in each pattern. Compared with the WAXS pattern of $0 \mathrm{Mrad}$ aPP, the scattering intensities of the principal peaks for the 400 and $1300 \mathrm{Mrad}$ iPP samples are lower by about $17 \%$ and $25 \%$, respectively. However, the peak positions are virtually unchanged in all WAXS patterns for the four samples.

The resultant DRDF curves for the aPP and iPP samples are shown in Figures 4 and 5, respectively. The DRDF for 0 Mrad sample consists of two intramolecular peaks at $\sim 1.5$ and $2.5 \AA$ and three intermolecular peaks at $6.6,13.0$, and $17.7 \AA$ (see APPENDIX). In addition to the two intramolecular peaks, the DRDF curve shows only two apparent intermolecular peaks at $\sim 7$ and $13 \AA$ when aPP is irradiated to $115 \mathrm{Mrad}$. Because of the error in-
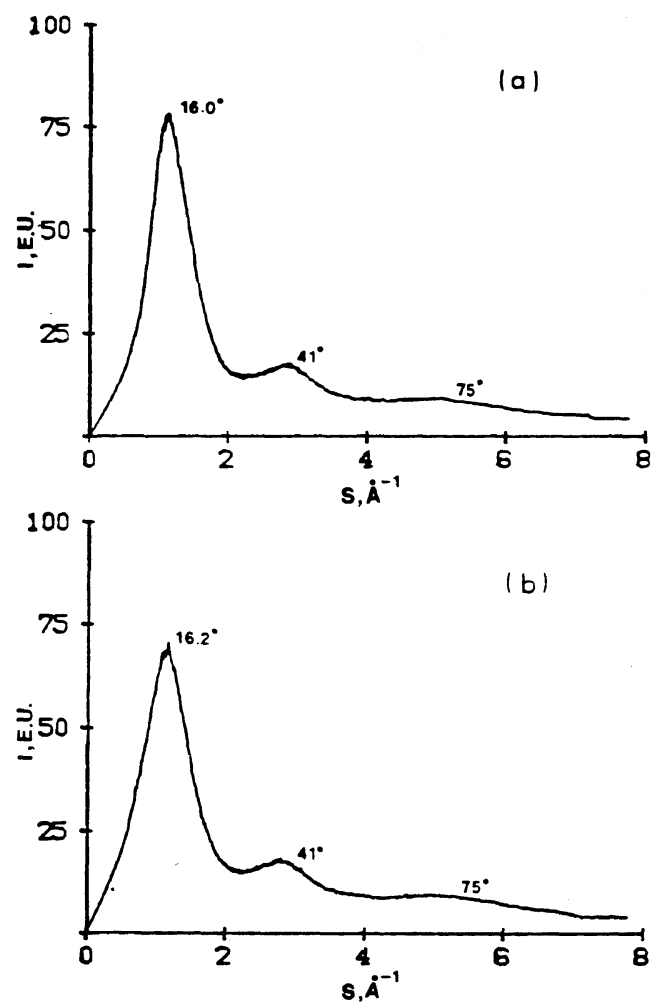

Figure 3. Corrected and normalized experimental WAXS data for (a) $400 \mathrm{Mrad}$ and (b) $1300 \mathrm{Mrad}$ isotactic polypropylene. The intensity data were normalized by the conservation of energy method. 

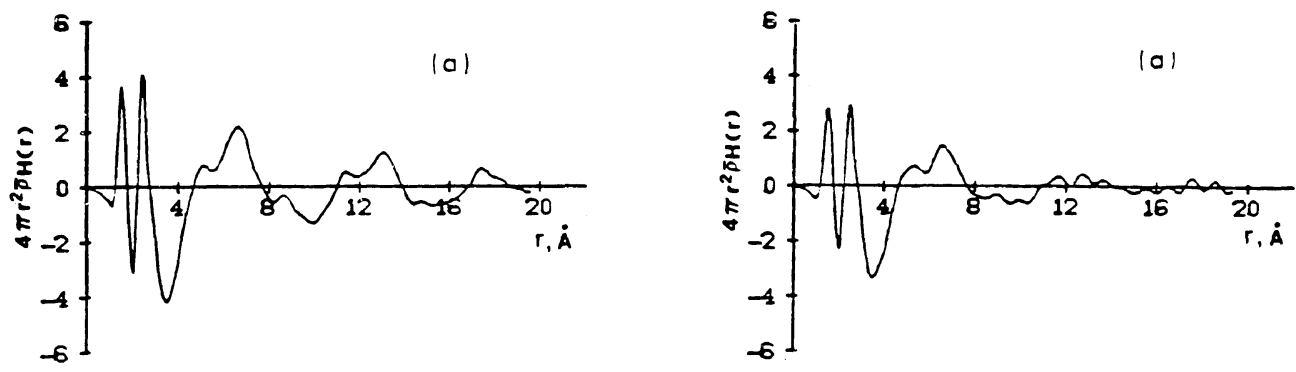

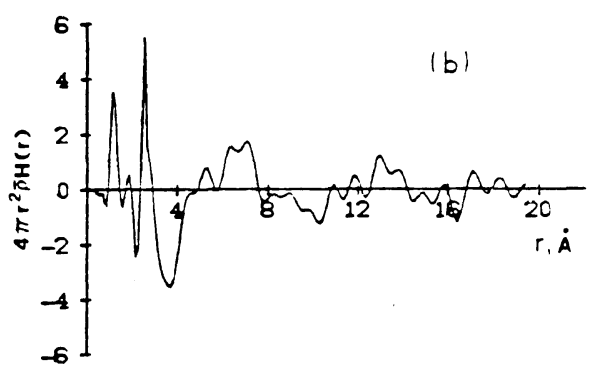

Figure 4. DRDF curves for atactic polypropylene: (a) $0 \mathrm{Mrad}$ and (b) $115 \mathrm{Mrad}$.

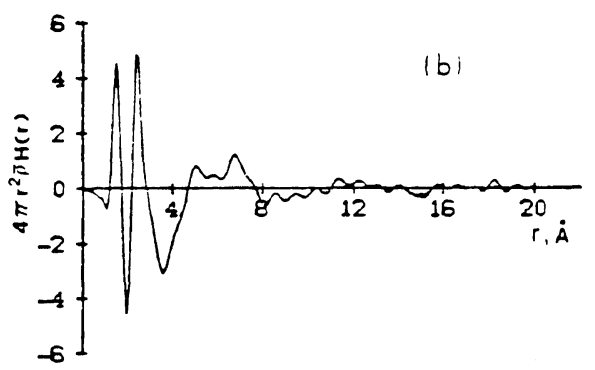

Figure 5. DRDF curves for radiation-amorphized isotactic polypropylene: (a) $400 \mathrm{Mrad}$ and (b) 1300 Mrad.

Table I. DRDF peak locations and areas for polypropylene

\begin{tabular}{|c|c|c|c|c|c|c|c|c|}
\hline \multirow{2}{*}{$\begin{array}{c}\text { Sample } \\
\text { peak }\end{array}$} & \multicolumn{2}{|c|}{0 Mrad aPP } & \multicolumn{2}{|c|}{$115 \mathrm{Mrad}$ aPP } & \multicolumn{2}{|c|}{$400 \mathrm{Mrad}$ iPP } & \multicolumn{2}{|c|}{$1300 \mathrm{Mrad}$ iPP } \\
\hline & Location & Area & Location & Area & Location & Area & Location & Area \\
\hline 1 & $1.31-1.83 \AA$ & 1.19 & $0.97-1.51$ & 1.07 & $1.23-1.81$ & 1.09 & $1.23-1.77$ & 1.52 \\
\hline 2 & $2.26-2.83$ & 1.21 & $2.36-2.92$ & 1.30 & $2.26-2.87$ & 1.07 & $2.23-2.74$ & 1.83 \\
\hline 3 & $4.71-7.76$ & 3.42 & $4.76-7.67$ & 2.63 & $4.67-7.69$ & 2.36 & $4.72-7.71$ & 1.72 \\
\hline 4 & $11.06-13.95$ & 1.89 & $11.55-14.23$ & 1.13 & - & - & - & - \\
\hline 5 & $16.87-18.78$ & 0.72 & - & - & - & - & - & - \\
\hline
\end{tabular}

troduced in collecting the WAXS data, more ripples are present in this DRDF curve.

For the radiation-amorphized iPP samples, pronounced effects of radiation on the intermolecular DRDF peaks can be' seen. The DRDF curves for 400 and 1300 Mrad iPP samples each show only one prominent intermolecular peak at $\sim 7 \AA$ in addition to the two commonly observed intramolecular peaks at 1.5 and $2.5 \AA$.

The results are summarized in Table I, which shows the location and area for all DRDF peaks.

\section{DISCUSSION}

For the 0 Mrad aPP sample, the DRDF curve showed an approximately constant peaking interval of $6 \AA$ for the intermolecular peaks at $6.6,13.0$, and $17.7 \AA$. Since the DRDF peaks represent deviation in atomic concentration from perfect homogenity, the apperarance of these periodic intermolecular peaks suggests the presence of more-or-less aligned parallel chains. A short-range structure having-a size of $\sim 18 \AA$ therefore existed in the unirradiated amorphous aPP. The $6 \AA$ periodicity may result from the average chain-to-chain distance between 


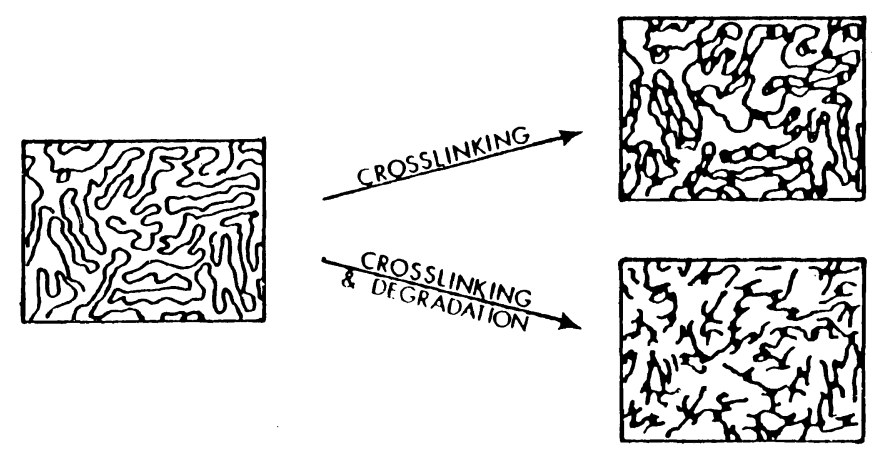

Figure 6. Effects of crosslinking and crosslinking combined with degradation on amorphous polymer structures.

such parallel molecules, as suggested by a recent analysis of the DRDF curve using Hosemann's paracrystalline lattice theory. ${ }^{13}$ Preliminary results of the analysis showed that the packing of chain segments in amorphous aPP was described reasonably well by a highly distorted hexagonal paracrystalline lattice.

The high-energy radiation causes a significant decrease in the intensity of intermolecular WAXS peaks $\left(\sim 16^{\circ} 2 \theta\right)$, as shown in Figures 2 and 3. The significance of these changes can better be understood by examining the intermolecular peaks of the resultant DRDF curves. As seen from Table I, the high-energy irradiation resulted in a decrease in the area of the intermolecular DRDF peaks, indicating that deterioration of intermolecular organization occurred in the irradiated polymers. This decrease in the area of DRDF peaks results in the elimination of higher ordered intermolecular peaks, or in the diminution of local structure size. For $115 \mathrm{Mrad}$ aPP, a local structure of $\sim 13 \AA$ was found owing to the existence of two intermolecular DRDF peaks remaining after radiation-induced deterioration. Similarly, severe deterioration of the intermolecular organization occurred in the amorphous PP samples as suggested by the single intermolecular peaks in the DRDF curves for 400 and 1300 Mrad iPP. It should be pointed out that DRDF curves consisting of only one intermolecular peak have not been commonly observed in amorphous polymers.

Furthermore, it is noted that high-energy radiation seems to cause structure deterioration more effectively in PP than in NR and PE. For aPP, as mentioned above, the third intermolecular DRDF peak at $17.7 \AA$ disappeared as a result of $115 \mathrm{Mrad}$ irradiation. Under the same irradiation conditions, structure deterioration of NR was not observed until the doses were higher than $300 \mathrm{Mrad}$. Also, the deterioration caused no size decrease in the local structure up to $550 \mathrm{Mrad}^{4} \mathrm{~A}$ similar comparison can be made for reactor irradiated iPP and PE. In contrast to the single intermolecular DRDF peak observed for $400 \mathrm{Mrad}$ iPP sample, five periodic peaks were seen up to $20 \AA$ for PE irradiated to the same dose. ${ }^{5}$ This different behavior may be attributed to the different radiation chemistry of the polymers, i.e., a combination of crosslinking and degradation in PP works different from crosslinking in NR and PE on deterioration of the local structure, as shown in Figuer 6. The schematic representation of the unirradiated amorphous polymers is shown on the left based on the structural model proposed by Yeh. ${ }^{14}$ For NR and PE, the existence of parallel chain segments is shown along with the radiation-induced crosslinks in the upper right of the figure. The local structure is no longer present in the irradiated PP as shown in the lower right of the figure.

Local structure deterioration resulted from the radiation-induced increase in the lattice distortion. ${ }^{4,6}$ Nagasawa and Kobayashi ${ }^{15}$ suggest that radiation-induced crosslinks shift adjacent chains in crystalline PE. This shift affects the molecular segments at a certain distance from the crosslinks resulting in lattice distortions. In consideration of this, lattice distortion in NR and amorphous PE should not be expected to increase very much, at least at the early stage of irradiation. The influence of the crosslinking-induced molecular shift on the originally highly distorted lattice in amorphous state is relatively insignificant compared to the crystalline lattice. For PP, the situation is different 
since the irradiated polymer undergoes substantial degradation in addition to crosslinking. The crosslinking-induced molecular shift is aided by degradation which cuts molecules into smaller segments. The lattice distortion therefore increases rapidly, destroying the intermolecular organization.

\section{CONCLUSION}

The DRDF study shows the existence of a local structure of $\sim 18 \AA$ in unirradiated aPP. The structure is deteriorated to $\sim 13 \AA$ when aPP is irradiated to $115 \mathrm{Mrad}$ and is extremely limited in radiationamorphized iPP due to the occurrence of severe structure deterioration. The deterioration is attributed to the crosslinking-induced lattice distortion.

Also, radiation seems to deteriorate the intermolecular organization more effectively in PP than in NR and PE. This is explained by the fact that irradiated PP undergoes substantial degradation in addition to crosslinking.

\section{APPENDIX}

It is known that DRDF peaks originate from two types of atomic regularities, i.e., the intramolecular and intermolecular distances. The former regularity depends on the repeat unit along the polymer chain, and the latter may consist of atomic distances due to the molecular organization. The contribution of each of these distances can therefore be estimated using the conformational analysis of the polymer chain as described in detail elsewhere. ${ }^{7}$
In Table II, the results of such a conformational analysis for the $0 \mathrm{Mrad}$ aPP are given. Due to the practical difficulty in formulating a general aPP chain conformation, crystalline chain conformation of both $\mathrm{iPP}^{16}$ and syndiotactic polypropylene $(\mathrm{sPP})^{17}$ were employed to calculate the theoretical contribution of intramolecular distances to the radial distribution function (RDF), $4 \pi r^{2} \bar{\rho}[H(r)+1]$.

As shown in Table II, the first two peaks at 1.58 and $2.51 \AA$ primarily originate from the intramolecular $C_{1}-C_{2}$ and $C_{1}-C_{3}$ distances, respectively. Agreement in matching the positions of these two peaks is within $3 \%$. The tacticity of the polymer is not expected to affect the calculated results for these two intramolecular distances.

By averaging all the possible intramolecular distances between 4.7 and $7.8 \AA$, intramolecular contributions of $16 \%$ and $22 \%$ to the third peak at $6.55 \AA$ are found from the conformation of iPP and $\mathrm{SPP}$, respectively. For an actual aPP chain, the intramolecular contribution in this distance range is not expected to be much higher than about $20 \%$, based on the atomic density consideration. The peak is therefore mainly intermolecular in origin. For the higher ordered peaks at 13.0 and $17.7 \AA$, the contribution of intermolecular distances must be even higher since, as the radius increases, the experimental peak areas increase much faster than those of the calculated intramolecular contributions.

In addition, Table II shows that the prediction of the position of the third peak is more accurate by using the conformation of sPP $(3.7 \%)$ than that of

Table II. Comparison of experimental ând calculated intramolecular RDF peak areas for unirradiated atactic polypropylene

\begin{tabular}{|c|c|c|c|c|c|c|c|}
\hline \multicolumn{2}{|c|}{$\begin{array}{l}\text { Experimental } \\
\text { RDF peaks }\end{array}$} & \multicolumn{3}{|c|}{$\begin{array}{c}\text { Calculated intramolecular } \\
\text { peaks from iPP }\end{array}$} & \multicolumn{3}{|c|}{$\begin{array}{l}\text { Calculated intramolecular } \\
\text { peaks from sPP }\end{array}$} \\
\hline$r / \AA$ & $\begin{array}{l}\text { Area/ } \\
\text { atom }\end{array}$ & $r_{\mathrm{av}} / \AA$ & $\begin{array}{l}\text { Area/ } \\
\text { atom }\end{array}$ & Ratio $^{e}$ & $r_{\mathrm{av}} / \AA$ & $\begin{array}{l}\text { Area/ } \\
\text { atom }\end{array}$ & Ratio \\
\hline 1.58 & 1.89 & $1.54^{\mathrm{a}}$ & 2.00 & 1.06 & $1.54^{\mathrm{a}}$ & 2.00 & 1.06 \\
\hline 2.51 & 3.08 & $2.56^{\mathrm{b}}$ & 2.67 & 0.87 & $2.52^{\mathrm{b}}$ & 2.67 & 0.87 \\
\hline 6.55 & 59.27 & $6.15^{\mathrm{c}}$ & 9.33 & 0.16 & $6.31^{d}$ & 13.00 & 0.22 \\
\hline
\end{tabular}

a Average of all possible intramolecular $\mathrm{C}_{1}-\mathrm{C}_{2}$ distances.

b Average of all possible intramolecular $C_{1}-C_{3}$ distances.

c Average of all possible intramolecular $C_{1}-C_{5}$ to $C_{1}-C_{9}$ distances in $4.7<r<7.8 \AA$.

d Average of all possible intramolecular $C_{1}-C_{5}$ to $C_{1}-C_{11}$ distances in $4.7<r<7.8 \AA$.

e $\operatorname{Ratio}=\left(\right.$ Area $\left._{\text {calcd }}\right) /\left(\right.$ Area $\left._{\text {expt1 }}\right)$. 
iPP $(6.1 \%)$. This perhaps suggests that the sPP molecular conformation can better describe the true situation of aPP chains. It is noted that the conformation of atactic polystyrene and its $p$-halogen derivative can also be represented by their respective syndiotactic molecules according to a previous diffraction study by Katada. ${ }^{18}$

\section{REFERENCES}

1. D. O. Geymer, in "The Radiation Chemistry of Macromolecules," Vol. II, M. Dole, Ed., Academic Press, New York, 1973.

2. A. Charlesby, "Atomic Radiation and Polymers," Pergamon Press, Oxford, 1960.

3. A. Chapiro, "Radiation Chemistry of Polymeric System," Interscience, New York, 1962.

4. C. S. Wang and G. S. Y. Yeh, J. Macromol. Sci., Phys., to appear.

5. M. R. Gupta and G. S. Y. Yeh, J. Macromol. Sci., Phys., B16,. 225 (1979).
6. M. R. Gupta, Ph.D. Thesis, The University of Michigan, 1978.

7. C. S. Wang and G. S. Y. Yeh, J. Macromol. Sci., Phys., B15, 107 (1978).

8. J. A. Sauer, L. J. Merrill, and A. E. Woodward, $J$. Polym. Sci., 58, 19 (1962).

9. J. N. Tomlinson and D. E. Kline, J. Appl. Polym. Sci., 11, 1931 (1967).

10. H. P. Klug and L. E. Alexander, "X-Ray Diffraction Procedures," John Wiley, New York, 1974.

11. K. Furukawa, Rep. Prog. Phys., 25, 395 (1962).

12. S. M. Wecker, M.S. Thesis, Northwestern University, 1970.

13. C. S. Wang and G. S. Y. Yeh, unpublished work.

14. G. S. Y. Yeh, J. Macromol. Sci., Phys., B6, 465 (1972).

15. T. Nagasawa and K. Kobayashi, J. Appl. Phys., 41, 4276 (1970).

16. G. Natta and P. Corradini, Nuovo Cimento, Suppl., 15, No. 1, 40 (1960).

17. G. Natta, Makromol. Chem., 35, 94 (1960).

18. K. Katada, Acta Cryst., 16, 290 (1963). 